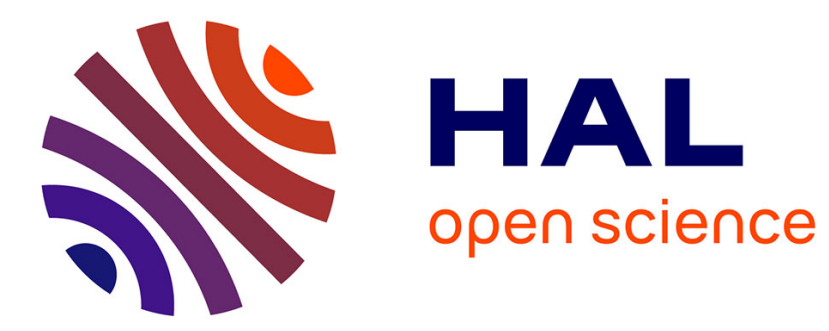

\title{
Le christ, le père noël et la laïcité, en France et aux États-Unis
}

Thomas Hochmann

\section{To cite this version:}

Thomas Hochmann. Le christ, le père noël et la laïcité, en France et aux États-Unis. Nouveaux cahiers du Conseil constitutionnel, 2016, pp.53-61. hal-01632362

\section{HAL Id: hal-01632362 \\ https://hal.science/hal-01632362}

Submitted on 10 Nov 2017

HAL is a multi-disciplinary open access archive for the deposit and dissemination of scientific research documents, whether they are published or not. The documents may come from teaching and research institutions in France or abroad, or from public or private research centers.
L'archive ouverte pluridisciplinaire HAL, est destinée au dépôt et à la diffusion de documents scientifiques de niveau recherche, publiés ou non, émanant des établissements d'enseignement et de recherche français ou étrangers, des laboratoires publics ou privés. 


\title{
Le christ, le père noël et la laïcité, en France et aux États-Unis
}

\author{
Thomas Hochmann \\ Professeur de droit public à l'Université de Reims Champagne-Ardenne
}

En dépit de la revue qui accueille ces pages, elles ne sauraient avoir pour objet de comparer la jurisprudence du Conseil constitutionnel et de la Cour suprême des États-Unis sur la question des relations entre l'État et la religion. Comme le soulignait récemment un membre du Conseil préfaçant un juge de la Cour suprême, on ne peut guère mettre sur le même plan les arrêts argumentés produits outre-Atlantique et les décisions du Conseil, dont la motivation «affirme plus qu'elle ne discute et ne démontre ». La « révolution» de la motivation qui serait en marche ${ }^{2}$ demeure trop récente et limitée pour contredire ce constat. En outre, le contrôle abstrait pratiqué par le Conseil ne lui permet de toutes façons pas d'être confronté à la variété de cas qui conduit la Cour suprême à forger et à affiner une jurisprudence.

Il n'est pas davantage possible de se livrer ici à une étude d'ensemble des multiples questions que pose la «laïcité » en France et aux États-Unis ${ }^{3}$. On abordera donc le sujet autrement, en concentrant le propos sur un problème particulier auquel ont été confrontées des juridictions en France et aux États-Unis. L'actualité française propose deux thèmes, qui ont chacun à voir avec une crèche. Le premier se révèle être une fausse piste. C'est en effet en termes de " laïcité » qu'est souvent traitée en France la question de l'interdiction du port du voile. Lorsqu'elle s'adresse comme ce fut récemment le cas à des personnes privées, une telle mesure ne concerne cependant pas la laïcité mais la liberté de religion. Les règles qui concrétisent la laïcité n'imposent d'obligation qu'aux autorités publiques et aux personnes qui agissent en leur nom ${ }^{4}$. Les nombreux arrêts de la Cour suprême qui touchent à ces questions, en particulier sous l'angle des exemptions religieuses qui doivent être accordées aux règles d'application générale ${ }^{5}$, sont donc hors de propos. Si l'on comprend la laïcité comme une exigence de neutralité des pouvoirs publics envers les religions, c'est une autre controverse récente qui s'avère pertinente. Au cours des dernières années, plusieurs juridictions administratives françaises sont parvenues à des conclusions opposées à propos des scènes de la nativité installées à Noël dans des bâtiments publics ${ }^{6}$. Or, c'est précisément le même problème qui a conduit la Cour suprême à préciser sa méthode de contrôle du respect par l'État de la neutralité religieuse. Il y a donc un intérêt certain à rappeler en France cette jurisprudence. Pour le dire vite, ce n'est pas la crèche de Baby Loup, mais celle de Baby Jesus, qui retiendra notre attention.

Le cadre juridique de la neutralité religieuse de l'État aux États-Unis doit être rappelé avant que soit examiné le problème particulier des crèches de Noël.

\footnotetext{
${ }^{1}$ Guy Canivet, « Préface », in Stephen Breyer, La Cour suprême, le droit américain et le monde, trad. fr. R. Brauchard, Odile Jacob, 2015, p. 15.

${ }^{2}$ Fanny Malhière, «Le considérant est mort! Vive les décisions du Conseil constitutionnel ? », Gazette du palais, 24 mai 2016, n 19. Cf. le communiqué du président du 10 mai 2016, www.conseil-constitutionnel.fr.

${ }^{3}$ Pour une présentation générale, cf. Arnaud Coutant, «La laïcité américaine, l'illusion démocratique », in Franck Laffaille (dir.), Laïcité(s), Mare et Martin, 2010, pp. 113-158; et Élisabeth Zoller (dir), La conception américaine de la laïcité, Dalloz, 2005.

${ }^{4}$ Sur cette question, cf. Stéphanie Hennette Vauchez et Vincent Valentin, L'affaire Baby Loup ou la nouvelle laïcité, LG.D.J., 2014 ; et V. Valentin, «Remarques sur les mutations de la laïcité. Mythes et dérives de la 'séparation' », RDLF 2016, chron. $\mathrm{n}^{\circ} 14$, www.revuedlf.com.

${ }^{5}$ Cf. Employement Division v. Smith, 494 U.S. 872 (1990) ; et récemment Burwell v. Hobby Lobby Stores, 573 U.S. _ (2014) ; Holt v. Hobbs, 574 U.S.__(2015).

${ }^{6} \mathrm{Cf}$. Mathieu Touzeil-Divina, «Trois sermons (contentieux) pour le jour de Noël. La crèche de la nativité symbole désacralisé : du cultuel au culturel ? », JCP A, n 23, 8 juin 2015, 1274 ; Mehdi Yazi-Roman, « Laïcité : la crèche de Noël dans l'enceinte des bâtiments des collectivités territoriales, emblème interdit d'une religion ou œuvre culturelle bienvenue? », AJ Collectivités Territoriales, 2015, p. 651.
} 


\section{L'interdiction américaine de l'« établissement » d'une religion}

En vertu du premier amendement à la Constitution des États-Unis, «Le Congrès ne fera aucune loi qui établirait une religion $»^{7}$. Selon la technique dite de $1^{\prime}$ " incorporation », il a été décidé que cette disposition, connue comme la «clause d'établissement», s'imposait également aux États fédérés. Il est donc interdit à toutes les autorités publiques « d'aider une religion, d'aider toutes les religions, ou de préférer une religion à une autre ${ }^{8}$. Plus concrètement, la Cour suprême expliqua en 1971 dans l'arrêt Lemon v. Kurtzman ${ }^{9}$ la manière dont il convenait de procéder pour vérifier le respect de cette règle (« Lemon test »). D'abord, la mesure litigieuse ne doit pas avoir un objectif religieux. Ensuite, son effet principal ne doit être ni de promouvoir ni d'inhiber la religion. Enfin, elle ne doit pas entraîner une implication excessive de l'autorité publique dans la religion ${ }^{10}$. Si la mesure litigieuse échoue à un seul de ces éléments, elle viole la clause d'établissement.

Le troisième élément, qui vise d'éventuels liens institutionnels entre l'État et la religion, peut être laissé de côté au profit des deux premiers critères, relatifs au but et aux effets de la mesure prise par l'autorité publique. Ces conditions, il faut le reconnaître, sont assez problématiques. Comment, par exemple, connaître le «but» poursuivi par l'État ? Dans une affaire relative à l'enseignement du créationnisme dans les écoles publiques, le juge Scalia s'était amusé de la vaine recherche de l'intention du législateur: peut-être les parlementaires qui avaient adopté la loi litigieuse souhaitaient-ils introduire la religion dans les cours de biologie. Mais le vote d'un individu pouvait tout aussi bien être aiguillé par des amitiés personnelles ou par une récente dispute avec sa femme. À supposer que l'on puisse identifier les motivations précises de chaque personne ayant voté pour la loi, combien d'entre elles doivent-elles être animées de l'intention inconstitutionnelle pour conclure à l'annulation de la loi litigieuse ? $^{11}$

Les questions posées par le «test Lemon » sont néanmoins parfaitement opératoires si elles sont envisagées d'une certaine manière. C'est à la juge O'Connor qu'il revient d'avoir, dans une série d'opinions individuelles, établi la méthode la plus convaincante pour contrôler le respect du premier amendement. Un acte étatique " établit» une religion s'il communique un message d'approbation de cette religion. Aussi la juge O'Connor envisage-t-elle la tâche du juge comme un exercice d'interprétation. Le premier amendement est enfreint si l'État souhaite communiquer un message d'adhésion à une religion ("intended message »), mais également si tel est le message effectivement communiqué, quand bien même l'État n'avait pas une telle intention («objective meaning $»)^{12}$. Ainsi O'Connor reformulait-elle les deux premières branches du test Lemon. La question pertinente se rapporte donc à la perception de l'acte étatique. Il s'agit de savoir si un « observateur objectif» et informé considérerait que la mesure litigieuse convoie le message selon lequel l'autorité publique adhère à une religion ${ }^{13}$.

\footnotetext{
${ }^{7}$ « Congress shall make no law respecting an establishment of religion [...]». Traduction d'Élisabeth Zoller, Les grands arrêts de la Cour suprême des États-Unis, Dalloz, 2010, p. 878.

${ }^{8}$ Everson v. Board of Education, 330 U.S. 1 (1947), p. 15.

9403 U.S. 602 (1971). Cf. É. Zoller, op. cit., pp. 397 ss.

${ }^{10}$ Lemon v. Kurtzman, cité, pp. 612 s. : «Every analysis in this area must begin with consideration of the cumulative criteria developed by the Court over many years. Three such tests may be gleaned from our cases. First, the statute must have a secular legislative purpose; second, its principal or primary effect must be one that neither advances nor inhibits religion; finally, the statute must not foster an excessive government entanglement with religion $»$ (citations omises).

${ }^{11}$ Edwards v. Aguillard, 482 U.S. 578 (1987), Scalia diss., pp. 636-639.

${ }^{12}$ Lynch v. Donnelly, 465 U.S. 668 (1984), O’Connor conc., p. 690.

${ }^{13}$ Wallace v. Jaffree, 472 U.S. 38 (1985), O’Connor conc., p. 76.
} 
Telle est l'essence du « test de l'adhésion » (endorsement test), qui fut ultérieurement retenu par la majorité de la Cour ${ }^{14}$.

Évidemment, l'interprétation de l'acte litigieux nécessite de tenir compte de son contexte : " tout comportement étatique doit être jugé dans ses circonstances particulières pour déterminer s'il constitue une adhésion [...] à une religion $»^{15}$. Une même mesure peut, en raison de circonstances divergentes, communiquer un message différent, et donc être conforme au premier amendement dans un cas mais pas dans l'autre. Les deux arrêts rendus le même jour en 2005 à propos des dix commandements forment un bon exemple. Dans une première décision, la Cour considérait qu'un monument sur lequel était inscrit le décalogue, qui était installé depuis quarante ans parmi d'autres statues sur le terrain qui entoure le capitole du Texas, n'était pas contraire à la Constitution ${ }^{16}$. La majorité était formée de deux opinions, la première réunissant quatre juges, et la seconde rédigée par Stephen Breyer. Bien que ces juges assuraient ne se référer ni au test Lemon ni au test de l'adhésion ${ }^{17}$, il semble bien que leur attention portait essentiellement sur le «message" communiqué par l'installation du monument litigieux, qui ne leur semblait pas essentiellement religieux, mais historique ou moral ${ }^{18}$. Le même jour, dans un autre arrêt, la Cour considérait en revanche que l'affichage des dix commandements dans un tribunal constituait, au vu du contexte dans lequel il était intervenu, une violation de la clause d'établissement ${ }^{19}$. La majorité était constituée des quatre juges dissidents dans Van Orden, ainsi que du juge Breyer. La Cour considéra que le «but» de l'autorité publique était religieux et échouait donc face au premier élément du test Lemon. Cependant, elle examina ce but du point de vue de l'« observateur raisonnable $»^{20}$. La constitutionnalité d'un bâtiment public d'apparence religieuse dépend donc de la question de savoir si, en vertu de son contexte historique, géographique et autre, il produit l'impression que la personne publique approuve le message religieux véhiculé. Ainsi, par exemple, un monument ancien, présent dans la commune depuis des dizaines d'années, sera moins susceptible d'être contraire au premier amendement: il fait en quelque sorte « partie du paysage » et ne transmet plus, ou plus uniquement, un message religieux ${ }^{21}$.

Le test de l'adhésion, comme le test Lemon, ont toujours été critiqués ${ }^{22}$. Récemment, une courte majorité de la Cour a exigé davantage qu'un message d'adhésion à une religion pour conclure à une violation du premier amendement. La mesure litigieuse doit exercer une contrainte, une pression d'un degré suffisant sur les administrés ${ }^{23}$. Mais la méthode de contrôle suggérée demeure semblable : l'existence d'une contrainte suffisante sera identifiée à l'aide du standard de $l^{\prime}$ ' observateur raisonnable $»^{24}$. Ainsi, plusieurs juges considèrent que la prière collective en ouverture d'un conseil municipal est admissible : à la lumière de la

\footnotetext{
${ }^{14}$ Santa Fe Independent School District v. Doe, 530 U.S. 290 (2000), p. 308.

${ }^{15}$ Lynch v. Donnelly, 465 U.S. 668 (1984), O’Connor conc., p. 694.

${ }^{16}$ Van Orden v. Perry, 545 U.S. 677 (2005).

${ }^{17}$ Cf. ibid., Breyer conc., pp. 699 s.

${ }^{18}$ Van Orden v. Perry, cité, p. 690 ; ibid., Breyer conc., p. 701.

${ }^{19}$ McCreary County v. ACLU of Kentucky, 545 U.S. 844 (2005).

${ }^{20}$ Ibid., pp. 866, 869 ; ibid., O'Connor conc., p. 883. Dans son opinion concordante jointe à Van Orden, le juge décisif dans ces deux arrêts, Stephen Breyer, précisa qu'il partageait la méthode d'analyse exprimée par la juge O’Connor dans McCreary County (Van Orden v. Perry, cité, Breyer conc. p. 698). Or, dans cette opinion, la juge O'Connor réitérait le "test» qui avait ses faveurs : l'administration viole la clause d'établissement si elle convoie le message d'une adhésion à une religion. Cf. McCreary County v. ACLU of Kentucky, cité, O'Connor conc., pp. 883 s.

${ }^{21}$ Cf. Van Orden v. Perry, cité, Breyer conc., pp. 702 s.

${ }^{22}$ Cf. par exemple Wallace v. Jaffree, 472 U.S. 38 (1985), Rehnquist diss., pp. 108 ss. ; County of Allegheny v. ACLU, 492 U.S. 573 (1989), Kennedy conc. et diss., p. 692 ; Lamb's Chapel v. Center Moriches Union Free School District, 508 U.S. 384 (1993), Scalia diss., p. 398 ;

${ }^{23}$ Town of Greece v. Galloway, 572 U.S. __ (2014).

${ }^{24} \mathrm{Cf}$. déjà en ce sens la réponse de la juge O'Connor au juge Kennedy, qui défendait une méthode de contrôle fondée sur la recherche d'une coercition, County of Allegheny v. ACLU, 492 U.S. 573 (1989), O'Connor conc., p. 629.
} 
tradition des prières législatives, les participants comprennent qu'il s'agit uniquement de conférer de la solennité à la réunion. Si un citoyen refuse d'y prendre part, son silence ne sera interprété ni comme un manque de respect, ni comme un accord avec les mots prononcés ${ }^{25}$. Dans le même sens, la juge O'Connor considérait que la devise "In God We Trust » imprimée sur la monnaie américaine, ou encore la pratique consistant à ouvrir les sessions de la Cour suprême par la formule "God save the United States and this honorable court", n'étaient pas perçues comme une approbation publique de certaines croyances, en raison de l'histoire et du caractère très répandu de ces pratiques ${ }^{26}$.

Cet examen en contexte des actes étatiques à connotation religieuse a d'abord été forgée par la juge O'Connor à propos des crèches de Noël. Ce raisonnement est-il pertinent pour résoudre le même problème dans le cadre juridique français?

\section{Neutralité et nativité}

L'arrêt Lynch v. Donnelly, rendu en 1984, portait sur une installation réalisée par la municipalité dans un quartier commercial. Une crèche était notamment accompagnée de la maison du père noël, d'un renne tirant un traineau, d'un sapin, mais également d'un éléphant, d'un clown et d'un ours en peluche ${ }^{27}$. Pour la juge O'Connor, dès lors que la crèche était incluse dans cet ensemble, elle ne pouvait être perçue comme une adhésion au christianisme. Il s'agissait plutôt de "célébrer une fête par ses symboles traditionnels ». On ne saurait, comme l'avait fait les juridictions inférieures, analyser la crèche indépendamment de l'ensemble de l'installation ${ }^{28}$. Cinq ans plus tard, la Cour se prononçait sur une crèche installée à l'intérieur d'un tribunal et surmontée d'un ange qui tenait dans ses mains l'inscription " Gloria in Excelsis Deo ». La majorité des juges, dont O'Connor, percevait là un message d'adhésion étatique au christianisme ${ }^{29}$.

C'est donc l'analyse contextuelle de la crèche qui permet de déceler ou d'écarter une adhésion inconstitutionnelle à des convictions religieuses. Les juridictions françaises récemment confrontées aux crèches installées dans des bâtiments publics ont suivi un raisonnement sensiblement différent. L'enjeu, pour elles, semble consister à savoir si la crèche est ou non un symbole religieux. Ainsi, une cour d'appel explique qu' " une crèche de Noël, dont l'objet est de représenter la naissance de Jésus, installée au moment où les chrétiens célèbrent cette naissance, doit être regardée comme ayant le caractère d'un emblème religieux », et en déduit que son installation dans un bâtiment public est illégale ${ }^{30}$. Dans la même affaire, le tribunal administratif de Melun avait au contraire considéré que, "si une crèche peut être regardée comme une reproduction figurative de la naissance de Jésus, elle est dépourvue de toute signification religieuse lorsque elle est installée temporairement en dehors des lieux de culte à l'occasion de la fête de Noël et hors de tout contexte rappelant la religion chrétienne, et constitue alors une des décorations traditionnellement associées à Noël comme le sapin de Noël ou les illuminations $»^{31}$. Dans le même sens, la cour administrative d'appel de Nantes juge que la crèche "s'inscrit dans le cadre d'une tradition relative à la préparation de la fête familiale de Noël et ne revêt pas la nature d'un "signe ou emblème religieux" ${ }^{32}$.

\footnotetext{
${ }^{25}$ Town of Greece v. Galloway, 572 U.S. _ (2014).

${ }^{26}$ Lynch v. Donnelly, 465 U.S. 668 (1984), O'Connor conc., p. 693 ; County of Allegheny v. ACLU, 492 U.S. 573 (1989), O’Connor conc., p. 630.

${ }^{27}$ Lynch v. Donnelly, 465 U.S. 668 (1984), p. 671.

${ }^{28}$ Ibid., O’Connor conc., p. 691.

${ }^{29}$ County of Allegheny v. ACLU, 492 U.S. 573 (1989), O’Connor conc. pp. 626 s.

${ }^{30}$ CAA Paris, , 8 octobre 2015, Fédération départementale des libres penseurs de Seine-et-Marne, AJCT 2015, 651.

31 TA Melun, 22 décembre 2014, Fédération départementale des libres penseurs de Seine-et-Marne, $\mathrm{n}^{\circ}$ 1300483. Nous soulignons.

${ }^{32}$ CAA Nantes, 13 octobre 2015, Département de la Vendée, AJCT 2015, p. 651.
} 
Bien sûr, affirmer que la représentation de la naissance miraculeuse du christ n'est pas un symbole religieux n'est guère convaincant ${ }^{33}$. Même entourée d'un renne et d'un sapin, la signification religieuse de la crèche ne saurait être éliminée ${ }^{34}$. La Cour suprême n'a jamais contesté cette évidence ${ }^{35}$. Simplement, elle considère que l'installation d'un symbole religieux n'exprime pas forcément une adhésion de l'État au culte concerné. Lorsqu'un tableau religieux est exposé dans un musée, expliquait la juge O'Connor, il ne perd pas sa signification religieuse. Simplement, son exposition ne transmet pas un message d'adhésion du musée à la religion. De même, la signification religieuse de la crèche n'est pas neutralisée lorsqu'elle figure à côté du père noël, mais l'ensemble modifie la perception raisonnable des observateurs. L'installation des symboles traditionnels de cette fête n'est généralement pas perçue comme une adhésion à la foi ${ }^{36}$.

Parmi les juridictions françaises, il en est une qui a retenu un raisonnement similaire. L'argumentation du tribunal administratif de Montpellier mérite d'être citée in extenso: la « crèche constitue l'exacte reproduction figurative de la scène de la naissance de Jésus de Nazareth, telle qu'elle est décrite dans l'évangile selon Luc ; qu'ainsi elle a une signification religieuse parmi la pluralité de significations qu'elle est susceptible de revêtir ; que, cependant, l'installation de cette crèche dans l'hôtel de ville a constamment été présentée, que ce soit auprès du conseil municipal, du préfet ou du public, comme une exposition s'inscrivant dans le cadre d'animations culturelles organisées à l'occasion des fêtes de Noël dans le coeur de ville, sans qu'aucun élément du dossier ne vienne révéler une intention différente et/ou la manifestation d'une préférence pour les personnes de confession chrétienne, au détriment du reste de la population ; qu'ainsi, dans les circonstances de l'espèce, l'installation de cette crèche ne peut être regardée comme ayant le caractère d'une présentation revendiquée de symboles de la religion chrétienne ${ }^{37}$.

Cette argumentation s'inscrit parfaitement dans la lignée de la jurisprudence américaine. Le tribunal recherche si l'installation de la crèche traduit une adhésion de la commune au christianisme ${ }^{38}$. Le problème est que le tribunal de Montpellier est chargé de contrôlé le respect de la loi française, et non de la Constitution américaine. Or, le droit français n'organise pas un régime semblable à celui que la Cour suprême a déduit du premier amendement. Il ne se limite pas à défendre aux autorités publiques de donner l'impression de favoriser une religion. L'article 28 de la loi du 9 décembre 1905 interdit beaucoup plus largement «d'élever ou d'apposer aucun signe ou emblème religieux sur les monuments publics ou en quelque emplacement public que ce soit». Le contexte semble donc indifférent : toute installation par la personne publique d'un symbole religieux contrevient à cette disposition, peu importe s'il ne semble pas traduire une adhésion à la foi. C'est probablement ce qui explique que certaines juridictions, auxquelles la solution imposée par la loi semblait sans doute " excessive ${ }^{39}$, aient été conduites à dénier le caractère religieux de la crèche. La loi française est beaucoup plus stricte que le régime américain : toute installation

\footnotetext{
${ }^{33}$ Cf. les critiques de Mathieu Touzeil-Divina, « Trois sermons (contentieux) pour le jour de Noël. La crèche de la nativité symbole désacralisé : du cultuel au culturel ? », JCP A, n 23, 8 juin 2015, 1274.

${ }^{34}$ Cf. Lynch v. Donnelly, 465 U.S. 668 (1984), Brennan diss., p. 708 : «I refuse to accept the notion implicit in today's decision that non-Christians would find that the religious content of the creche is eliminated by the fact that it appears as part of the city's otherwise secular celebration of the Christmas holiday. ».

${ }^{35}$ Lynch v. Donnelly, 465 U.S. 668 (1984), p. 685.

${ }^{36}$ Lynch v. Donnelly, 465 U.S. 668 (1984), O’Connor conc., p. 692.

${ }^{37}$ TA Montpellier, 16 juillet 2015, AJCT 2015, 651.

${ }^{38}$ Une autre question est de savoir si la conclusion négative à laquelle le tribunal parvient est correcte. Quiconque s'intéresse quelque peu au comportement du maire de la commune concernée peut avoir des doutes.

${ }^{39}$ Cf. en ce sens Lynch v. Donnelly, 465 U.S. 668 (1984), p. 686 : « To forbid the use of this one passive symbol -- the creche -- at the very time people are taking note of the season with Christmas hymns and carols in public schools and other public places, and while the Congress and legislatures open sessions with prayers by paid chaplains, would be a stilted overreaction contrary to our history and to our holdings ». Nous soulignons.
} 
publique d'un symbole religieux est en principe interdit, qu'il s'inscrive ou non dans un message d'adhésion de l'autorité publique à la foi.

Il existe pourtant une manière d'accepter en France certaines crèches sans, comme le tribunal de Montpellier, modifier la loi ni, comme le tribunal de Melun ou la cour de Nantes, dénaturer les faits. L'article 28 de la loi de 1905 prévoit en effet certaines exceptions qui concernent « [1] es édifices servant au culte, [1]es terrains de sépulture dans les cimetières, [1]es monuments funéraires, ainsi que [1] es musées ou expositions ». Le parallèle tracé par la juge O'Connor entre une peinture religieuse dans un musée et une crèche installée parmi les décorations de Noël nous met sur la voie. Il n'est sans doute pas impossible d'analyser l'installation de certaines crèches comme une "exposition». C'est d'ailleurs ainsi que le tribunal de Montpellier décrit l'installation litigieuse, même s'il ne fonde pas sa décision sur cette exception prévue par la loi ${ }^{40}$.

Ce terme d'« exposition » se prête à plusieurs interprétations qui permettent plus ou moins largement l'installation de crèches. L'interprétation la plus restrictive entend l'exposition dans un sens muséal. Seules seront alors autorisées les crèches composées de figurine d'une certaine valeur historique ou artistique, dignes d'être "placée[s] sous vitrine ${ }^{41}$. Une interprétation beaucoup plus large distingue le fait $d$ ' $"$ exposer » de l'action « d'élever ou d'apposer », interdite en vertu de l'article 28. La différence résiderait alors dans le caractère provisoire ou permanent de l'installation, et la crèche de Noël serait protégée. Une interprétaion médiane considère qu'un signe attaché à un culte est simplement « exposé » lorsque le message religieux n'est pas endossé par l'autorité publique. Constituerait alors une exposition l'installation décorative d'une crèche, entre guirlandes et sapins. L'analyse juridictionnelle, telle qu'elle fut menée à Montpellier, rejoindrait alors le raisonnement américain, dès lors qu'il s'agirait de savoir si la crèche traduit ou non une adhésion de la commune au christianisme.

L'adoption d'une grille d'analyse semblable en France et aux États-Unis ne conduira néanmoins pas forcément, pour des faits similaires, au même résultat dans les deux systèmes. En effet, cet examen juridictionnel est sensible au contexte, et celui-ci inclut, outre les circonstances propres à la crèche litigieuse, la perception plus générale de la religion dans une société. Jamais un juge français ne pourra vraisemblablement affirmer, comme la juge O'Connor, que la reconnaissance de la religion, par exemple sous la forme d'une prière au sein du parlement, est «la seule manière raisonnable dans notre culture de conférer de la solennité à certaines cérémonies publiques, d'exprimer de la confiance en l'avenir, et d'encourager la reconnaissance de ce qui est digne d'appréciation dans notre société ${ }^{42}$. Les places très différentes qu'occupe la religion en France et aux États-Unis devraient trouver une traduction dans l'appréciation juridictionnelle des faits. Peut-être que les tribunaux français, même s'ils retiennent une lecture de l'article 28 qui exempte de son application les crèches non prosélytes, seront moins enclins que les juges américains à identifier concrètement un tel cas de figure. Peut-être que certaines juridictions considéreront pourtant que la crèche, si elle a bien une signification religieuse, ne fait dans les circonstances de l'espèce que s'inscrire dans les décorations de Noël et ne traduit nulle adhésion publique au christianisme.

Ce qui n'est guère convaincant, c'est de défendre l'ineptie selon laquelle la scène de la nativité ne serait pas un symbole religieux. Plusieurs auteurs attendent du Conseil d'État qu'il mette de l'ordre dans la jurisprudence administrative sur cette question ${ }^{43}$. Mais ce rôle

\footnotetext{
${ }^{40}$ TA Montpellier, 16 juillet 2015, AJCT 2015, 651: «l'installation de cette crèche dans l'hôtel de ville a constamment été présentée, que ce soit auprès du conseil municipal, du préfet ou du public, comme une exposition s'inscrivant dans le cadre d'animations culturelles organisées à l'occasion des fêtes de Noël dans le coeur de ville ». Nous soulignons.

${ }^{41}$ Hélène Pauliat, « Crèches et bâtiments publics : la discorde », JCP A, n 49, 7 décembre 2015, act. 1002.

${ }^{42}$ Lynch v. Donnelly, 465 U.S. 668 (1984), O'Connor conc., p. 693.

${ }^{43}$ M. Touzeil-Divina, art. cit. ; M. Yazi-Roman, art. cit.
} 
pourrait aussi échoir au Conseil constitutionnel, à l'occasion d'une question prioritaire de constitutionnalité relative à l'article 28 de la loi de 1905. Peut-on imaginer plus belle occasion que la nativité pour que cette institution, dont on dit souvent qu'elle est née plusieurs fois ${ }^{44}$, adopte enfin des décisions précisément motivées, bref pour qu'elle naisse (encore une fois) à nouveau, qu'elle soit « born again », comme tout bon chrétien américain ?

${ }^{44}$ Cf. notamment Léo Hamon, Les juges de la loi, Fayard, 1987, p. 159. 